\title{
Silver nanoparticle toxicity in Drosophila: size does matter
}

\author{
This article was published in the following Dove Press journal: \\ International Journal of Nanomedicine \\ I0 February 201I \\ Number of times this article has been viewed
}

\author{
Deborah J Gorth' \\ David M Rand ${ }^{2}$ \\ Thomas J Webster' \\ 'School of Engineering, ${ }^{2}$ Department \\ of Ecology and Evolutionary Biology, \\ Brown University, Providence, RI, USA
}

Correspondence: Thomas J Webster School of Engineering, Brown University, Providence, RI 02912, USA

Email thomas_webster@brown.edu
Background: Consumer nanotechnology is a growing industry. Silver nanoparticles are the most common nanomaterial added to commercially available products, so understanding the influence that size has on toxicity is integral to the safe use of these new products. This study examined the influence of silver particle size on Drosophila egg development by comparing the toxicity of both nanoscale and conventional-sized silver particles.

Methods: The toxicity assays were conducted by exposing Drosophila eggs to particle concentrations ranging from $10 \mathrm{ppm}$ to $100 \mathrm{ppm}$ of silver. Size, chemistry, and agglomeration of the silver particles were evaluated using transmission electron microscopy, X-ray photoelectron spectroscopy, and dynamic light scattering.

Results: This analysis confirmed individual silver particle sizes in the ranges of 20-30 nm, $100 \mathrm{~nm}$, and 500-1200 nm, with similar chemistry. Dynamic light scattering and transmission electron microscope data also indicated agglomeration in water, with the transmission electron microscopic images showing individual particles in the correct size range, but the dynamic light scattering z-average sizes of the silver nanoparticles were $782 \pm 379 \mathrm{~nm}$ for the $20-30 \mathrm{~nm}$ silver nanoparticles, $693 \pm 114 \mathrm{~nm}$ for the $100 \mathrm{~nm}$ silver nanoparticles, and $508 \pm 32 \mathrm{~nm}$ for the 500-1200 nm silver particles. Most importantly, here we show significantly more Drosophila egg toxicity when exposed to larger, nonnanometer silver particles. Upon exposure to silver nanoparticles sized $20-30 \mathrm{~nm}$, Drosophila eggs did not exhibit a statistically significant $(P<0.05)$ decrease in their likelihood to pupate, but eggs exposed to larger silver particles (500-1200 nm) were $91 \% \pm 18 \%$ less likely to pupate. Exposure to silver nanoparticles reduced the percentage of pupae able to emerge as adults. At $10 \mathrm{ppm}$ of silver particle exposure, only $57 \% \pm 48 \%$ of the pupae exposed to 20-30 nm silver particles became adults, whereas $89 \% \pm 25 \%$ of the control group became adults, and $94 \% \pm 52 \%$ and $91 \% \pm 19 \%$ of the $500-1200 \mathrm{~nm}$ and $100 \mathrm{~nm}$ group, respectively, reached adulthood.

Conclusion: This research provides evidence that nanoscale silver particles $(<100 \mathrm{~nm})$ are less toxic to Drosophila eggs than silver particles of conventional $(>100 \mathrm{~nm})$ size.

Keywords: Drosophila, silver, nanoparticle, toxicity

\section{Introduction}

Nanotechnology, or the use of materials with one dimension less than $100 \mathrm{~nm}$, offers the ability to change particle reactivity by simply changing their size. This novel property of nanomaterials has been used to create more effective treatments for cancer, and improve tissue engineering and regenerative medicine. Nanoscale materials are also being used at an increasing rate in commercial products, with silver nanoparticles representing a sizable portion of the industry, thus it is essential to understand their potential toxicity, as well as the mechanism of their toxicity to be able to control 
their environmental impact. ${ }^{1,2}$ It is hypocritical to extol the enhanced reactivity and unique properties of nanoparticles for certain commercial applications, but not appreciate their potential enhanced toxicity to the environment due to these same properties. If nanoparticles are more reactive than conventional-sized particles, it is certainly plausible that they may also be more toxic.

Studying the environmental toxicity of silver nanoparticles is of particular interest because they are currently being used in a wide range of commercial ${ }^{3,4}$ and medical products ${ }^{5}$ like antibacterial clothing and wound dressings. Silver nanoparticles are by far the most common nanoscale chemical additive to consumer products and comprise over $50 \%$ of the nanotechnology consumer industry; of the 483 products inventoried as of August 25, 2009 by the Project on Emerging Nanotechnologies, 259 of those products contained silver nanoparticles. ${ }^{6}$ Of these products, over a third present a risk of releasing nanoscale silver into the environment. ${ }^{4}$ Because of these factors, a recent study labeled silver nanoparticle release into the environment a moderate to high ecotoxicological risk, about which we know very little. ${ }^{7}$

Most of the current research examining nanoparticle toxicity uses in vitro methods that do not factor in the tendency of a particle to accumulate in tissues or be cleared from a whole organism. ${ }^{8}$ The toxicity of a substance can be greatly affected by its propensity to accumulate in part of an organism, increasing the local concentration of the toxicant. Neglecting the organism by conducting in vitro analysis alone limits the accuracy of determining toxicity. The studies that do look at whole organisms have mostly focused on aquatic organisms, finding that nanoparticles have toxic effects in rainbow trout, zebra fish, Caenorhabditis elegans, algae, and daphnids. ${ }^{9-14}$ However, it is critical in all nanoparticle studies to accurately characterize the particle being studied and to make accurate comparisons with larger particles of the same chemistry, because only then will we know the impact of particle size (without chemical interferences) on toxicity.

This project explored the dependence of the nature and severity of the toxicity of silver nanoparticles per their size by exposing 50 Drosophila eggs to food sources with 10 or $100 \mathrm{ppm}$ of silver nanoparticles, and monitoring their development. To isolate the size-dependent nature of nanoparticle toxicity, this study used three different sizes of silver nanoparticles (20-30 nm, $100 \mathrm{~nm}$, and 500-1200 nm) and confirmed their similar chemistry using X-ray photoelectron spectroscopy. This is important because we know that chemistry greatly influences toxicity, and this study was designed to isolate the influence of particle size alone on toxicity. Once similar chemistry was established, dynamic light scattering and transmission electron microscopy were used to examine individual particle size and agglomeration tendencies.

This present study broadens the existing in vivo environmental toxicity research. Drosophila are a model organism representing a low-level terrestrial heterotrophic organism. They are present in many waterbed environments that may be exposed to silver nanoparticle waste. Therefore, the results of this research would provide critical information to begin to guide regulations for the industrial manufacturing and use of silver nanoparticles.

\section{Materials and methods Materials}

Silver particles with sizes in the 20-30 nm, $100 \mathrm{~nm}$, and 500-1200 nm ranges were purchased from SkySpring Nanomaterials (20-30 and $100 \mathrm{~nm}$; Houston, TX) and Inframat Advanced Materials (500-1200 nm; Manchester, CT), and were used without any further modification.

\section{Material analysis}

The size and agglomeration tendencies of these silver particles were evaluated using dynamic light scattering and transmission electron microscopy, using the same solution of an appropriate quantity of nanoparticles in water to approximate their distribution in water-based food, as described below. Transmission electron microscopic images were taken with a Philips/FEI CM20 transmission electron microscope operating at $200 \mathrm{keV}$. The chemistry of these particles was evaluated using X-ray photoelectron spectroscopy on a PHI 5500 Multitechnique Surface Analyzer (Multi-Tech Systems, Eden Praire, MN) using an Al K-alpha X-ray source with an energy of $1486.6 \mathrm{eV}$. The hydrodynamic diameters of the silver particles were measured using a Zeta Nano S-90 dynamic light scattering instrument (Malvern Instruments, Malvern, UK).

\section{Drosophila culture media production}

Yeast-based fly food and grape juice agar plates were both made prior to the onset of the experiment; $250 \mathrm{~mL}$ of yeast-based food consisted of water $200 \mathrm{~mL}$, agar $2.173 \mathrm{~g}$, SAF yeast $5.5 \mathrm{~g}$, cornmeal $14.3 \mathrm{~g}$, sugar $20.25 \mathrm{~g}$, and $20 \%$ of a Tegosept solution in ethanol $3.094 \mathrm{~mL}$. First, the agar was dissolved completely in the water, then all of the remaining ingredients, except for the Tegosept solution, were added to the mixture heated at $90^{\circ} \mathrm{C}$. After the ingredients were added and the mixture was allowed to thicken for 10 minutes, it was 
removed from the heat source. Once the food cooled below $80^{\circ} \mathrm{C}$, the Tegosept solution was added.

The grape juice agar plates were made using agar, Tegosept, grape juice, and distilled water. First, the agar $(50 \mathrm{~g})$ and water $(1500 \mathrm{~mL})$ were mixed and autoclaved. The mixture was then kept warm on a hotplate above $80^{\circ} \mathrm{C}$, and when the mixture cooled to under $80^{\circ} \mathrm{C}, 500 \mathrm{~mL}$ of grape juice and $30 \mathrm{~mL}$ of Tegosept (10\% in ethanol) were added.

\section{Organisms}

The flies used for these toxicity assays were the wild type Drosophila melanogaster strain Oregon $\mathrm{R}$ incubated at $25^{\circ} \mathrm{C}$ on a $7 \mathrm{am}$ to $7 \mathrm{pm}$ light cycle and a $7 \mathrm{pm}$ and $7 \mathrm{am}$ light cycle. Before toxicity assays were performed, the flies were transferred from a yeast-based food to the aforementioned grape juice agar plates with a small portion of yeast paste (dry active yeast wetted with deionized water) for use as a protein source for the laying flies. Twelve hours before fly eggs were to be used for the toxicity assays, the agar plates were changed insuring that all eggs were less than 12 hours old at the onset of the experiment.

\section{Toxicity assays}

The various toxicity treatments were added to the yeastbased food during initial food production. A large amount of untreated food was made but was heated to just below $80^{\circ} \mathrm{C}$, maintaining its liquid form. From this batch, the three control vials were drawn and allowed to set in regular Drosophila culture vials. Enough food for the three replicate vials, ie, $30 \mathrm{~mL}$, was then pipetted from the untreated mixture into a secondary flask containing the nanoparticles for one toxicity treatment. Prior to being incorporated with the food, appropriate weights of silver nanoparticles were dissolved in $1 \mathrm{~mL}$ of water and then sonicated for 30 seconds to encourage suspension. The food and toxicity treatments were then mixed using a stir bar for 10 minutes before being allocated to three fly vials, $10 \mathrm{~mL}$ each. Food was allowed to set overnight. Fifty fly eggs under 12 hours old, cultured as already mentioned, were manually transferred to each treatment, and a control of yeast-based food without added particles was used. Egg pupation was determined by counting the pupae adherent to the sides of the Drosophila culture vials. Egg maturation was determined by counting the flies that completely emerged from their pupae.

\section{Atomic absorption spectrometry}

To determine the amount of silver accumulation after exposure to silver particles, atomic absorption spectrometry was used to quantify the silver concentration in the adult Drosophila. Three Drosophila per silver treatment (20-30 nm at $10 \mathrm{ppm}, 100 \mathrm{~nm}$ at $10 \mathrm{ppm}, 500-1200 \mathrm{~nm}$ at $10 \mathrm{ppm}$, and no treatment) and two blank vials were weighed, then treated with $1 \mathrm{~mL}$ of nitric acid for 20 hours in a $37.5^{\circ} \mathrm{C}$ shaker. Once fully digested, the nitric acid was evaporated on a hotplate. The digested tissue was then suspended in $2 \%$ nitric acid for atomic absorption. The silver content of the samples was measured using a PerkinElmer Analyst 600 atomic absorption spectrometer, with absorption measured using a $328.1 \mathrm{~nm}$ bulb corresponding to silver absorption. Silver standards of $0 \mathrm{ppb}, 10 \mathrm{ppb}, 20 \mathrm{ppb}, 30 \mathrm{ppb}, 40 \mathrm{ppb}$, and $50 \mathrm{ppb}$ were used to calibrate the absorption spectrometer and develop a nonlinear fit line with an $\mathrm{R}^{2}$ value $>0.99$. For analysis, $20 \mu \mathrm{L}$ of samples were dispensed for each analysis. If the silver concentration of this sample size exceeded the concentration of the calibration solution, $6 \mu \mathrm{L}$ of the sample was dispensed and diluted by the PerkinElmer Analyst 600 (PerkinElmer, Waltham, MA) with $2 \%$ nitric acid to achieve a total sample volume of $20 \mu \mathrm{L}$. If the concentration of the sample was still too high, $2 \mu \mathrm{L}$ of the sample was combined with $2 \%$ nitric acid until the total volume reached $20 \mu \mathrm{L}$ for analysis. Once the dilution procedure achieved an acceptable concentration of the sample for analysis, the PerkinElmer Analyst measured the silver absorption and then repeated the same dilution procedure; so two measurements of the absorption for the sample were taken for each sample.

\section{Statistics}

All experiments were conducted in triplicate. Statistics were completed using the Student's $t$-test. $P$ values $<0.05$ were considered statistically significant.

\section{Results \\ Particle analysis}

All three particle sizes tended to agglomerate, as indicated by both the transmission electron microscope images and dynamic light scattering results. The $20-30 \mathrm{~nm}$ sample was bimodal, with one peak ranging from $18.7-37.84 \mathrm{~nm}$ and a second peak from 122.4-190.1 nm and a z-average diameter of $782 \pm 379 \mathrm{~nm}$, indicating a diverse population of agglomerated particles. The $100 \mathrm{~nm}$ sample had one broad peak ranging from $141.8-342 \mathrm{~nm}$ and a z-average value of $693 \pm 114 \mathrm{~nm}$. The $500-1200 \mathrm{~nm}$ sample had a peak ranging from $164.2-531.2 \mathrm{~nm}$ and a z-average value of $508 \pm 32 \mathrm{~nm}$ (Figure 1D). The transmission electron microscopy results confirmed that the silver nanoparticles agglomerated, but the individual particles were within the pre- 


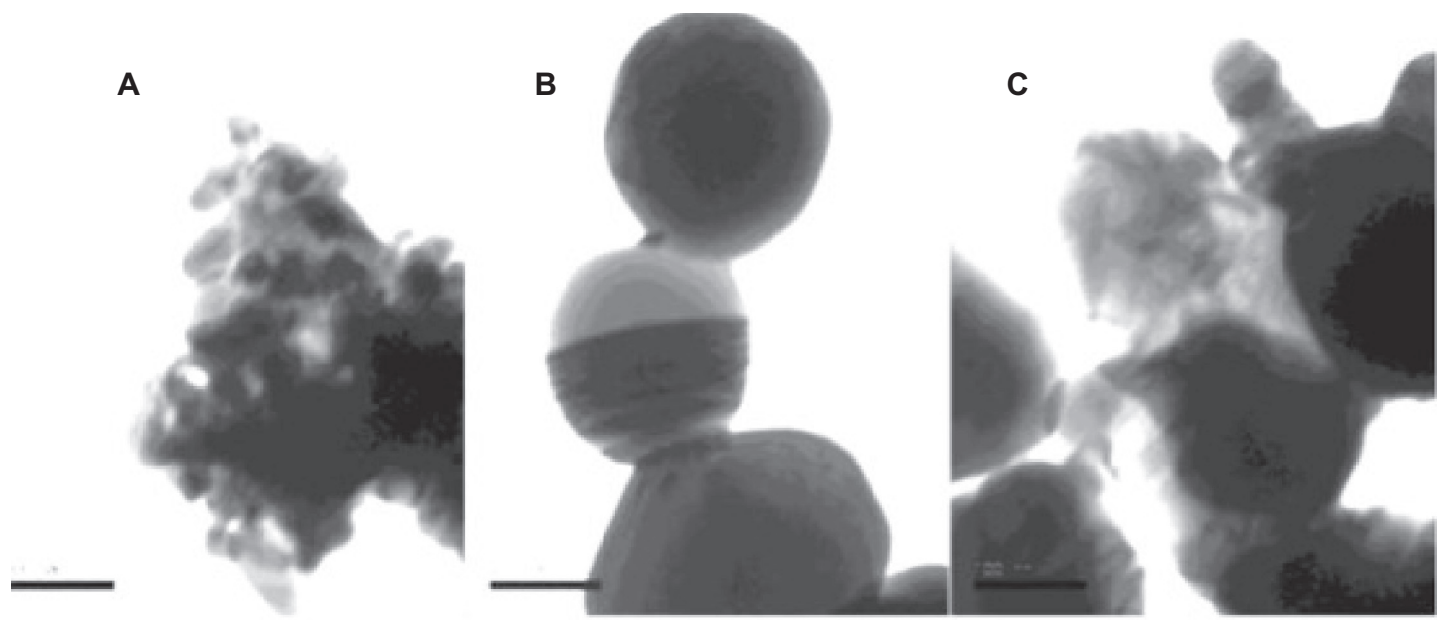

D

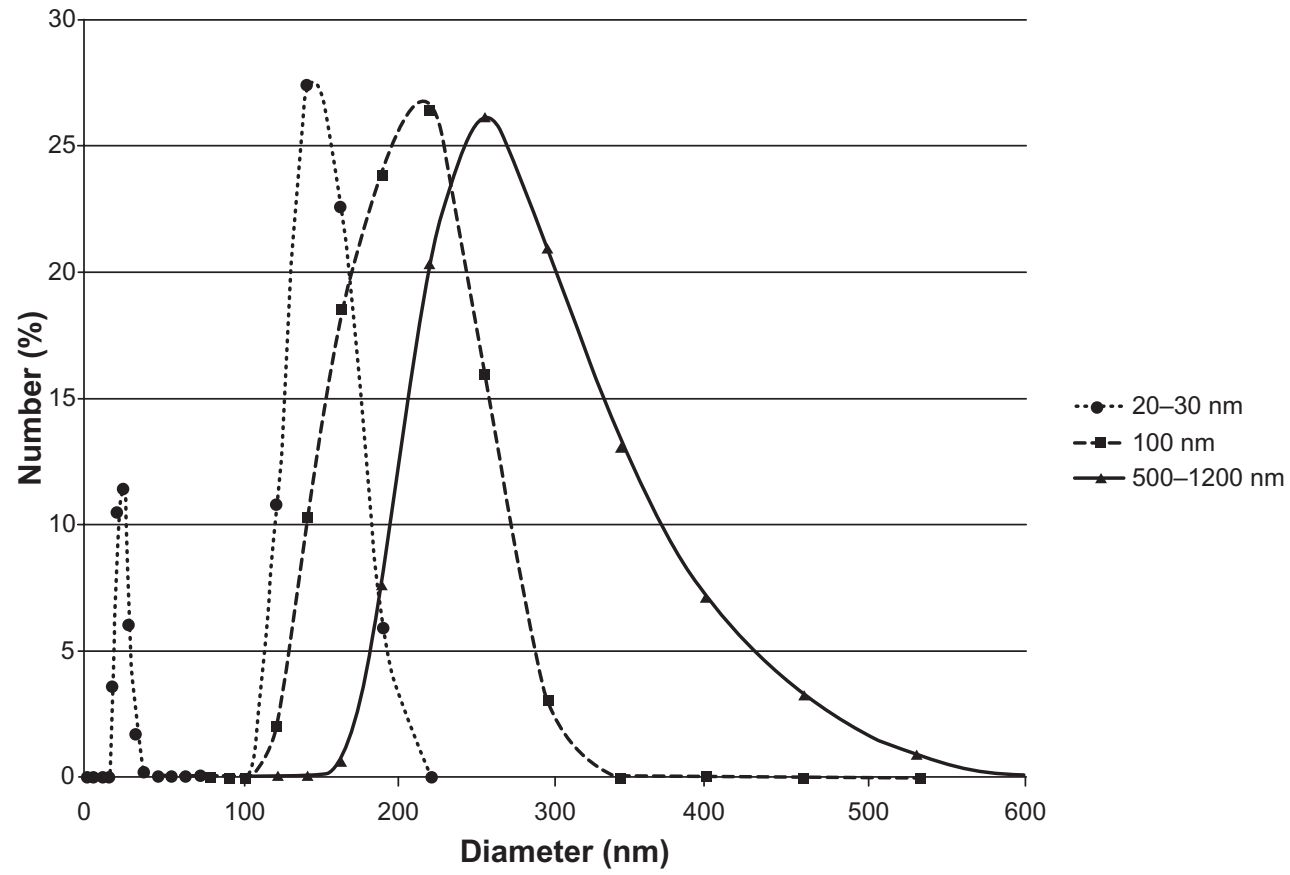

Figure I Particle characterization. Transmission electron microscopy images of A) 20-30 nm, B) $100 \mathrm{~nm}$, and C) 500-1200 nm silver particles. D) Dynamic light-scattering results for the silver nanoparticles.

dicted ranges (Figures 1A, 1B, and 1C). X-ray photoelectron spectroscopy data confirmed the chemical similarities between the three sizes of particles. Figure 2 shows the X-ray photoelectron spectroscopy data for the three sizes overlaid onto one grid. Although the $\mathrm{X}$-ray photoelectron spectroscopy data confirmed that all three silver particles were of the same chemistry, the dynamic light scattering and transmission electron microscopy data suggested that the advertized sizes of industrially fabricated nanoparticles did not correspond directly to the actual size of the purchased particles. The aim of this study was to work with silver particle toxicity in conditions most similar to accidental environmental exposure of industrially produced silver nanoparticles, so despite the poor quality of the silver particles used, they are of different sizes and representative of silver particles used in industrial applications today.

\section{Toxicity assays}

Overall, the $20-30 \mathrm{~nm}$ and $100 \mathrm{~nm}$ silver nanoparticles were significantly less toxic to the Drosophila larvae than the larger 500-1200 nm particles. At $10 \mathrm{ppm}$, the nanoscale silver particles $(100 \mathrm{~nm}$ and $20-30 \mathrm{~nm})$ had a statistically $(P<0.05)$ indistinguishable effect on the ability of Drosophila larvae to pupate as compared with each other and the control; 


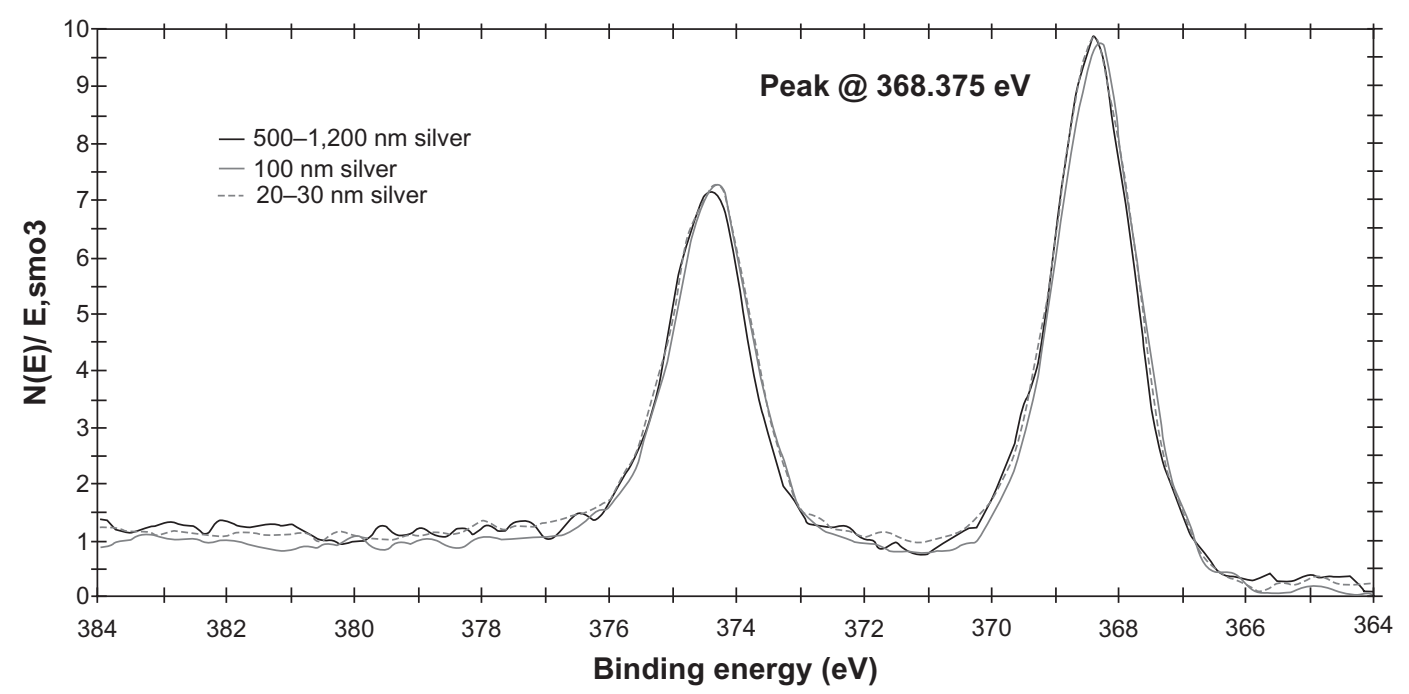

Figure 2 X-ray photoelectron spectroscopy data for the three sizes of silver nanoparticles, 500-1200 nm silver (black), $100 \mathrm{~nm}$ silver (grey), $20-30 \mathrm{~nm}$ silver (dotted).

$59 \% \pm 10 \%$ of the initial 50 eggs exposed to the $100 \mathrm{~nm}$ particles and $47 \% \pm 15 \%$ of the eggs exposed to $20-30 \mathrm{~nm}$ particles were able to pupate. Of the 50 eggs seeded in the control, $59 \% \pm 10 \%$ were able to pupate. In contrast with this result, only half as many eggs $(34 \% \pm 12 \%)$ were able to develop into pupae when exposed to the larger 500-1200 nm silver particles (Figure 3A).

The silver particles were more toxic in the Drosophila development stage between pupation and larval emergence. Although the $20-30 \mathrm{~nm}$ and $100 \mathrm{~nm}$ sizes did not affect the ability of the larvae to pupate, the results of this study did show that exposure of the eggs to the silver nanoparticles reduced the percentage of pupae that were able to emerge as adults. At 10 ppm of silver particle exposure, only $57 \% \pm 48 \%$ of the pupae exposed to 20-30 nm silver particles became adults, whereas $89 \% \pm 25 \%$ of the control group became adults, and $94 \% \pm 52 \%$ and $91 \% \pm 19 \%$ of the $500-1200 \mathrm{~nm}$ and $100 \mathrm{~nm}$ group, respectively, reached adulthood. However, at higher concentrations, the pupae exposed to $20-30 \mathrm{~nm}$ particles were more likely $(13 \% \pm 4 \%)$ than those exposed to larger particles (500-1200 nm, $0 \% \pm 0 \%$; and $100 \mathrm{~nm}, 5 \% \pm 1 \%$ ) to reach adulthood.
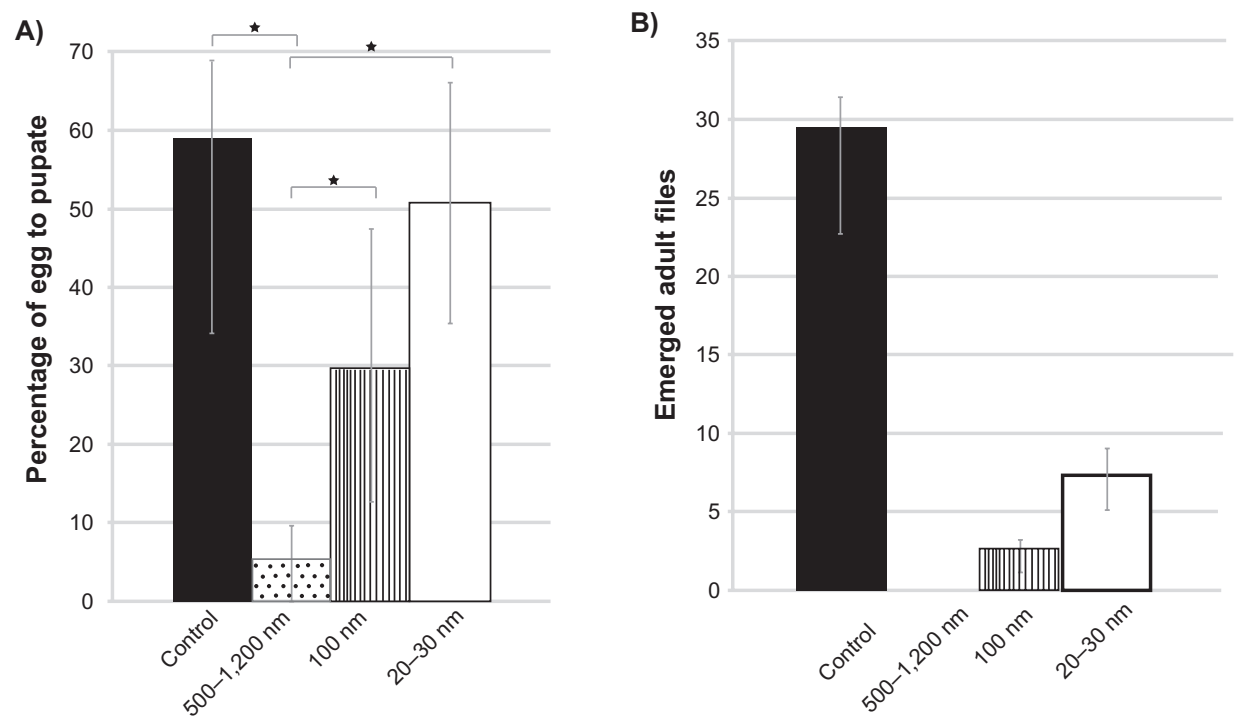

Figure 3 The in vivo toxicity of silver nanoparticles toward Drosophila eggs. Data expressed as mean \pm standard deviation. A) The percentage of Drosophila eggs able to pupate when exposed to no silver particles (black), 500-1200 nm silver particles at 100 ppm (dots), $100 \mathrm{~nm}$ silver particles at 100 ppm (stripes), or 20-30 nm silver particles at 100 ppm (white). $* P<0.05$. B) Number of adult flies able to emerge from 50 eggs exposed to no silver particles (blue), 100 ppm of $500-1200$ nm silver particles (red), 100 $\mathrm{Ppm}$ of $100 \mathrm{~nm}$ particles (green), or $100 \mathrm{ppm}$ of $20-30 \mathrm{~nm}$ particles (purple). All values are statistically different $(P<0.05)$. Data is mean \pm one standard deviation, $\mathrm{n}=3$. 
Despite reducing the likelihood that pupae would emerge, the 20-30 nm silver particles were less toxic to the overall developmental success of the Drosophila eggs. At 100 ppm, the success of full Drosophila development followed the same trend as the success of pupation. Figure 3B shows this trend. It should also be noted that when the Drosophila eggs were exposed to 20-30 nm silver nanoparticles, they emerged with reduced pigmentation, suggesting that exposure to silver nanoparticles did have effects more subtle than organism toxicity which needs to be further studied in the future. An image comparing a representative Drosophila exposed to $20-30 \mathrm{~nm}$ silver nanoparticles with a control is shown in Figure 4.

\section{Concentration-dependent toxicity}

As expected, the results of this study also showed that higher silver particle concentrations accentuated the size-dependent toxicity toward Drosophila eggs. The percentage of eggs exposed to the 20-30 nm silver particles that developed into pupae $(51 \% \pm 15 \%)$ remained indistinguishable from the control $(59 \% \pm 9.9 \%)$ even at $100 \mathrm{ppm}$, but when the concentration of the $100 \mathrm{~nm}$ and 500-1200 nm treatments increased to $100 \mathrm{ppm}$, the viability of the Drosophila eggs dropped from $59 \% \pm 10 \%$ to $30 \% \pm 17 \%$ and from $34 \% \pm 12 \%$ to $5 \% \pm 4 \%$, respectively. The silver particle

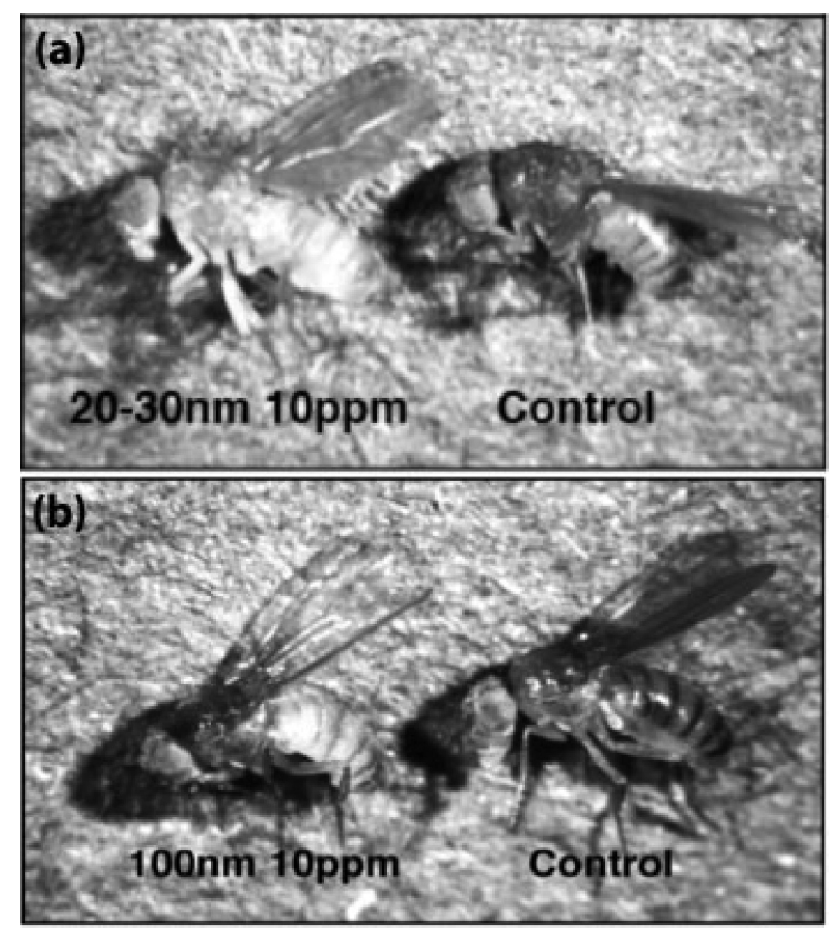

Figure 4 Light microscopy images of Drosophila exposed to 20-30 nm silver nanoparticles at $10 \mathrm{ppm}$ and no nanoparticles (A), and a Drosophila exposed to $100 \mathrm{~nm}$ silver particles at $10 \mathrm{ppm}$ and no nanoparticles (B). concentration-dependent toxicity for the $100 \mathrm{~nm}$ and 500-1200 nm particles suggests that increasing the exposure concentration leads to an increase in the biologically active concentration in the organism. The lack of an increase in egg development toxicity for the smaller 20-30 nm particles is evidence that smaller particles are metabolized or dealt with differently compared with larger silver particle sizes, and that an increase in exposure concentration does not directly translate to an increase in the experienced toxic dose for silver nanoparticles (20-30 nm). Figure 5 shows these results.

\section{Silver concentration in emerged adults}

The amount of silver in the Drosophila tissue exposed to the various toxicity treatments was measured using atomic absorption as described in the Methods section. The concentration of silver was only measured in the Drosophila exposed to $10 \mathrm{ppm}$ of the silver particles, because exposure to $100 \mathrm{ppm}$ of silver particles resulted in complete lethality for the eggs exposed to 50-1200 nm particles, so there were no Drosophila exposed to 500-1200 nm at this concentration for comparison. For the concentration assay, three Drosophila from each replicate vial were digested in nitric acid before being resuspended for the concentration measurement. Two blank vials were subjected to the same treatment as the Drosophila vials to subtract out any background noise in the final calculation of silver concentration.

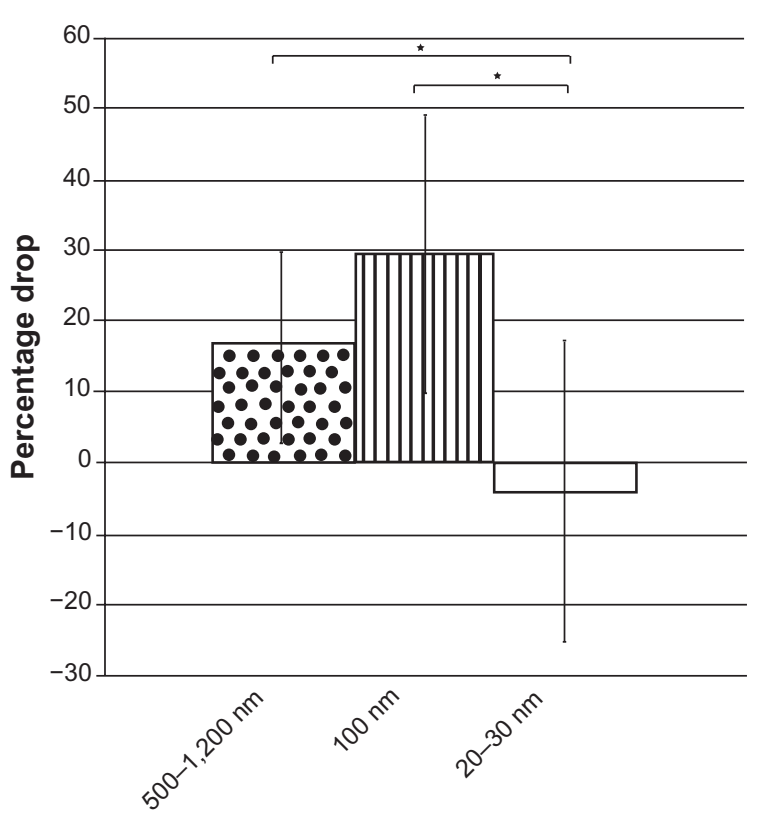

Figure 5 The percentage drop in eggs able to develop into pupae after increasing the exposure concentration from 10 ppm to 100 ppm; 500-1200 nm silver particles (dots), $100 \mathrm{~nm}$ silver nanoparticles (stripes), and 20-30 nm silver particles (white). Notes: ${ }^{*} p<0.05$. Data are expressed as mean \pm standard deviation, $n=3$. 
After subtracting out the concentration of silver in the blank vial and standardizing the silver concentrations to the weights of the flies, $0 \pm 0.00091 \mu \mathrm{g}$ of silver/g of Drosophila tissue was found in the control flies, $385.64 \pm 20.19 \mu \mathrm{g}$ of silver/g of Drosophila was found in the Drosophila exposed to the 20-30 nm nanosilver, $69.03 \pm 0.97 \mu \mathrm{g}$ of silver/g of Drosophila tissue was found in the Drosophila exposed to $100 \mathrm{~nm}$ nanosilver and $14.17 \pm 0.17 \mu \mathrm{g}$ of silver/g of Drosophila tissue was found in the flies exposed to $500-1200 \mathrm{~nm}$ particulate silver. Figure 6 summarizes these results. The scale of the difference between the amounts of silver found in the Drosophila exposed to the smaller particles comes to light when the data are shown graphically. As expected, there was no silver found in the control, but the eggs that were more likely to pupate, ie, those exposed to $20-30 \mathrm{~nm}$ particles, also resulted in flies with a much higher concentration of silver.

\section{Discussion}

This study provides the first evidence that silver nanoparticles may be less toxic than their conventionally-sized counterparts of identical chemistry for Drosophila egg development. This result is superficially antithetical to the traditional view of nanoscale reactivity, ie, the smaller a particle becomes, the more surface area per unit volume exposed to the organism to react. It would logically follow that if a substance had a high level of toxicity, reducing its size would consequently

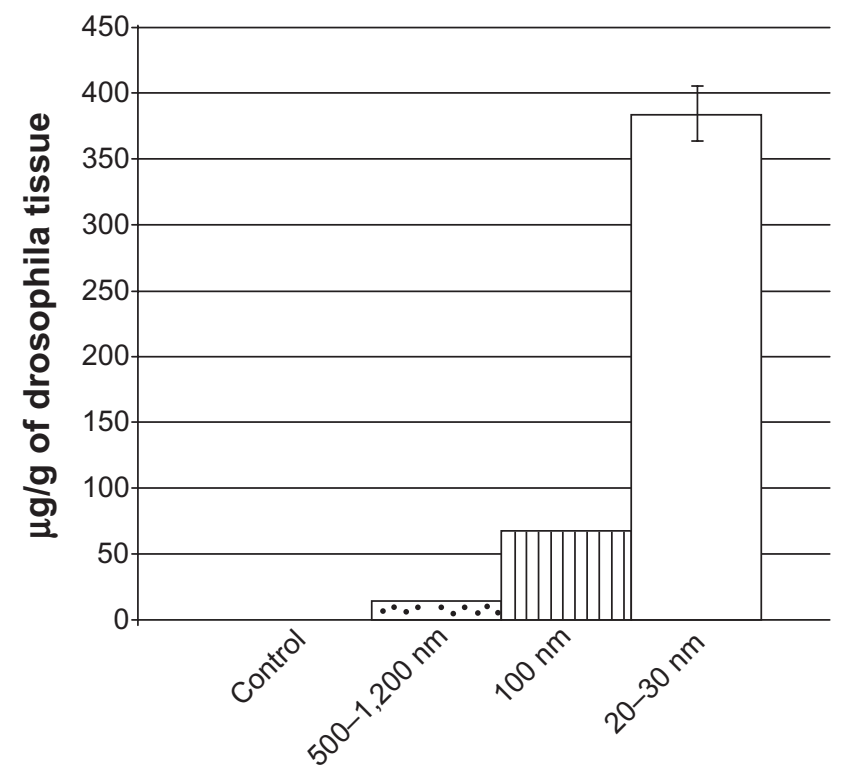

Figure 6 The silver concentration measured in samples of Drosophila tissue with the control with none, Drosophila exposed to $10 \mathrm{ppm}$ of $20-30 \mathrm{~nm}$ particles in plane, Drosophila exposed to $10 \mathrm{ppm}$ of $100 \mathrm{~nm}$ particles in bars, and Drosophila exposed to $10 \mathrm{ppm}$ of $500-1200 \mathrm{~nm}$ in dots. All values are statistically different from each other $(P<0.0 \mathrm{I})$ and data are presented as means, and error bars represent one standard deviation, $\mathrm{n}=3$. increase its reactivity per unit mass to increase the potential for toxicity. The unique reactivity of nanoscale silver particles is at least partially due to the release of silver ions, which readily occurs on the surface of nanoscale silver, and the smaller the particle, the more surface area that is exposed to the environment for ion release. ${ }^{15}$ Cytotoxicity experiments examining the effects of silver exposure have shown an increase in toxicity as silver nanoparticles decrease in size, and attributed this size-dependent difference to increased silver ion release. ${ }^{16}$ Despite this fact, the $20-30 \mathrm{~nm}$ silver particles were less toxic to developing Drosophila eggs than the larger 500-1200 nm silver particles. Although this result is unexpected, it does not contradict current research. Factors like bioaccumulation and biolocalization can also affect toxicity, and size can also play a role in how particles behave in vivo.

Recent studies have shown size-dependent localization and accumulation of nanoscale particles. Lankveld et al demonstrated less silver particle accumulation in all organs evaluated from Wistar rats (liver, lungs, spleen, brain, heart, kidneys, and testes) when injected with $20 \mathrm{~nm}$ silver than both $80 \mathrm{~nm}$ or $110 \mathrm{~nm}$ particles. ${ }^{17}$ Based on this information alone, it would follow that because fewer $20 \mathrm{~nm}$ silver particles accumulated than did both the $80 \mathrm{~nm}$ and $110 \mathrm{~nm}$ silver particles, they would also be less toxic.

In addition to the difference in organism used by Lankveld et al and the present study, another major difference is the route of particle exposure. In the present study, the silver particles were ingested as opposed to injected into the organism. This difference could account for the differences in bioaccumulation, with the $20-30 \mathrm{~nm}$ particles accumulating at a higher concentration in the currently presented ingestion study and $20 \mathrm{~nm}$ silver particles accumulating less in the injection study. When injected, membrane permeability can increase renal clearance, but when ingested, increasing membrane permeability can increase absorption. Despite the increased accumulation of the $20-30 \mathrm{~nm}$ silver particles as compared with the $100 \mathrm{~nm}$ and $500-1200 \mathrm{~nm}$ particles, differential biolocalization could account for the observed differences in toxicity with the $20-30 \mathrm{~nm}$ particles accumulating in nonessential locations, rendering them inert. Despite not causing lethality in Drosophila, the increase in silver concentration in Drosophila tissue exposed to $20-30 \mathrm{~nm}$ silver particles compared with both the $100 \mathrm{~nm}$ silver particles and the 500-1200 nm silver particles could make the smaller particles more toxic to higher trophic levels.

Although requiring further study, the lack of an increase in toxicity for the $20-30 \mathrm{~nm}$ silver particles suggests that the 
particles did not accumulate as readily inside the vital organs of the organism as did both the $100 \mathrm{~nm}$ and 500-1200 nm particles, potentially leading to a reduction in the delivered toxicity dose for the 20-30 nm particles. Future studies should further quantify the bioaccumulation of silver nanoparticles to verify if differential particle localization accounts for the reduced toxicity of 20-30 nm silver nanoparticles. Moreover, future studies are needed to continue to determine the effect of silver nanoparticle exposure on Drosophila development.

\section{Conclusion}

This study showed a size-dependent toxicity of silver particles to Drosophila development. The smaller silver nanoparticles, sized 20-30 $\mathrm{nm}$, had less of an effect than both $100 \mathrm{~nm}$ and $500-1200 \mathrm{~nm}$ silver particles on the ability of Drosophila eggs to develop into pupae and adult Drosophila. Exposure to 20-30 nm particles did not exhibit a concentration-dependent effect when the concentration increased from $10 \mathrm{ppm}$ to $100 \mathrm{ppm}$, but increasing the exposure dosage of the $100 \mathrm{~nm}$ and 500-1200 nm silver particles resulted in a corresponding decrease in larva and pupa viability. This research adds to the growing body of knowledge suggesting that, in addition to concentration, size has an influence on silver particle toxicity.

\section{Acknowledgment}

The research was funded by the Herman Foundation.

\section{Disclosure}

The authors report no competing financial interests in this work.

\section{References}

1. Colvin VL. The potential environmental impact of engineered nanomaterials. Nat Biotechnol. 2003;21:1166-1170.
2. Auffan M Bottero JY, Lowry GV, Jolivet J-P, Wiesner MR. Towards a definition of inorganic nanoparticles from an environmental, health and safety perspective. Nat Nanotechnol. 2009;4:634-641.

3. Kulthong K, Boonpavanitchakul K, Kangwansupamonkon W, Maniratanachote R. Determination of silver nanoparticle release from antibacterial fabrics into artificial sweat. Part Fibre Toxicol. 2010;7: $25-35$.

4. Luoma SN. Silver nanotechnologies and the envionment: Old problems or new challenges? Woodrow Wilson International Center for Scholars. 2008;3:67-79.

5. Chaloupka K. Nanosilver as a new generation of nanoproduct in biomedical applications. Trends Biotechnol. 2010;23:1-9.

6. The Project on Emerging Nanotechnologies, 2010. Available from: http://www.nanotechproject.org/inventories/consumer/analysis_draft/. Accessed September 25, 2010.

7. O'Brien CE. Ranking initial envionmental and human health risk resulting from environmentally relevant nanomaterals. J Environ Sci Health A Tox Hazard Subst Environ Eng. 2010;45:992-1007.

8. Xiaoke H, Wang P, Hwang H. In vitro evaluation of cytotoxicity of engineering metal oxide nanoparticles. Science of the Total Environment. 2009;407:3070-3072.

9. Scown TM, Johnston BD, Gaiser B, et al. Effects of aqueous exposure to silver nanoparticles of different sizes in rainbow trout. Toxicol Sci. 2010;115:521-534.

10. Wang $\mathrm{H}$, Xing B. Toxicity of nanoparticulate and bulk $\mathrm{ZnO}, \mathrm{Al}_{2} \mathrm{O}_{3}$ and $\mathrm{TiO}_{2}$ to the nematode Caenorhabditis elegans. Environ Pollut. 2009;157: 1171-1177.

11. Simon KH. Ecotoxic effect of photocatalytic active nanoparticles $\left(\mathrm{TiO}_{2}\right)$ on algae and daphnids. Environ Sci Pollut Res Int. 2004;124: 225-232.

12. Bouldin JL, Sengupta A, Alexander R, Hannigan R, Buchanan RA. Aqueous toxicity and food chain transfer of quantum dots (TM) in freshwater algae and ceriodaphina dubia. Environ Toxicol Chem. 2008; 27:1958-1963.

13. Navarro E, Wagner B, Marconi F, et al. Toxicity of silver nanoparticles to Chlamydomonas Reinhardtii. Envion Sci Technol. 2008;42: 8959-8964.

14. Asharani PV, Gong Z, Valiyaveettil S. Toxicity of silver nanoparticles in zebrafish models. Nanotechnology. 2008;19:2551002.

15. Liu J. Ion release kinetics and particle persistence in aqueous nano-silver colloids. Environ Sci Technol. 2010;44:2169-2175.

16. Carlson C, Hussain SM, Schrand AM, et al. Unique cellular interaction of silver nanoparticles: Size-dependent generation of reactive oxygen species. J Phys Chem B. 2008;11:13608-13619.

17. Lankveld D, Krystek P, Neigh A, et al. The kinetics of the tissue distribution of silver nanoparticles of different sizes. Biomaterials. 2010;31: $8350-8361$.
International Journal of Nanomedicine

\section{Publish your work in this journal}

The International Journal of Nanomedicine is an international, peerreviewed journal focusing on the application of nanotechnology in diagnostics, therapeutics, and drug delivery systems throughout the biomedical field. This journal is indexed on PubMed Central, MedLine, CAS, SciSearch $\AA$, Current Contents ${ }^{\circledR} /$ Clinical Medicine,

\section{Dovepress}

Journal Citation Reports/Science Edition, EMBase, Scopus and the Elsevier Bibliographic databases. The manuscript management system is completely online and includes a very quick and fair peer-review system, which is all easy to use. Visit http://www.dovepress.com/ testimonials.php to read real quotes from published authors. 